\title{
Measurements of Transport Properties on HTSC under Uniaxial Stress
}

\author{
N.Watanabe, T.Nakajima, N.Takahashi and K.Tsushima* \\ Gunma National College of Technology, 580 Toriba-Machi, Maebashi, Gunma 371, Japan \\ *Aso Fukuoka Junior College, 6-3-1 Saifu, Dazaifu, Fukuoka 818-01, Japan
}

\begin{abstract}
Measuring systems of transport properties, such as electrical conductivity, AC susceptibility and Hall coefficient, on High $T_{c}$ Superconductors (HTSC) under uniaxial stress are presented. The accuracy of them are confirmed to be sufficient for our study on HTSC, and they are applied for Bi2212 single crystal. The result which was obtained from measurements under uniaxial stress revealed that a simple charge transfer model between $\mathrm{BiO}$ and $\mathrm{CuO}_{2}$ planes is not sufficient for the system.

[HTSC, uniaxialstress, anisotropy, Halleffect, AC susceptibility]
\end{abstract}

\section{Introduction}

After 10 years studies on HTSC, much more precise studies on their properties are required. In HTSC, stress is applied to several purpose. One is the synthesis under high pressure. Also, stress effect on transport properties of HTSC has been studied so extensively. For instance, the highest $T_{c}$ of $165 \mathrm{~K}$ is obtained by applying stress for $\mathrm{Hg}$ system, which has $T_{c}$ of $134 \mathrm{~K}$ under ambient condition[1]. One of the most remarkable feature of HTSC is their high anisotropy of transport properties, reflecting the structure. However, measurements under uniaxial stress has rarely been done so far except several reports in La-[2][3] and Y-[4] systems, probably because of the difficulty of the measurements.

In the previous papers[5][6][7], we compared the dependence of the coefficient $d T_{c} / d P$ on hydrostatic and that on uniaxial stress by measuring electrical condutivity. In this paper, apparatus for measurements of transport properties under uniaxial stress are introduced, and the totally different results from that under hydrostatic stress are presented.

\section{Experimental Procedure}

The measurements of transport properties in the vicinity of $T_{c}$ under uniaxial stress have several difficulties as follows;

1) Maximum uniaxial stress is usually much smaller than that of hydrostatic case by one order of magnitude. Typically it is $2 \mathrm{kbar}$ or so, while hydrostatic stress can be applied up to several tens GPa.

2)Therefore, the change of the properties is much smaller in uniaxial case.

3)Temperature should be controlled much carefully near by the $T_{c}$. We should control it within $0.1 \mathrm{~K} / \mathrm{min}$. As for resolution of temperature, $10 \mathrm{mK}$ or less is required.

4)In the case of hydrostatic stress, organic fluid is usually used as stress transmitting medium. In the case of uniaxial stress, a pair of rods/disks is used for it. The uniformity of uniaxial stress should be confirmed.

When we apply uniaxial stress to the specimen, it is quite difficult to construct an adiabatic system, since stress transmitting media is a set of rod/disk, not vacuum. On the other hand, uniformity of temperature within the specimen should be ensured. A pair of sapphire rods or disks are used for our system, since they have high thermal conductivity and low electrical conductivity. Acrylic and copper blocks are employed to keep temperature of a sample room to be uniform.

\subsection{Electrical Conductivity}

Fig. 1 and 2 show the apparatus for electrical conductivity under uniaxial stress. Stress is applied onto the specimen which is sandwiched by a pair of sapphire disks. The surface of the specimen was polished to be parallel within the accuracy of $1 \mathrm{deg}$. To ensue the uniformity of stress, a small amount of resin is pasted onto the interface of the disks and the specimen. Measurements on electrical conductivity near by $T_{c}$ is a good method to check uniformity of the stress. When stress is applied not parallel to the specimen, a broadening of the transition region of superconductivity is observed since an electrical conductivity is sensitive for the path of the conduction. In our measurements, uniaxial stress was applied uniformly, since no broadening was observed.

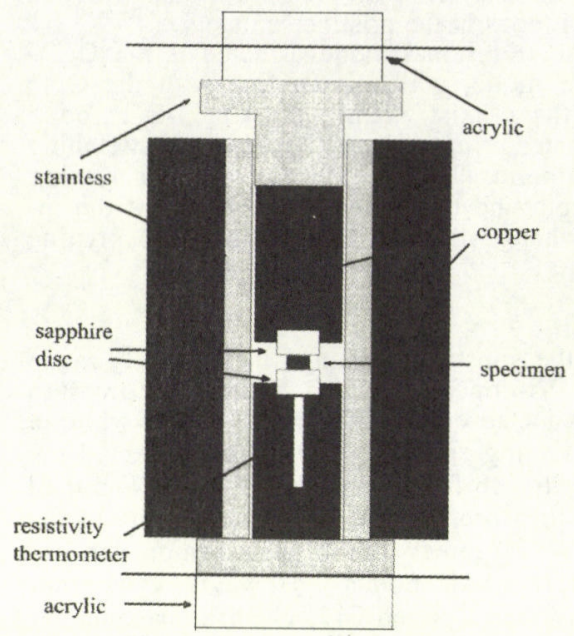

Fig.1. Scheme of the apparatus for electrical conductivity. 


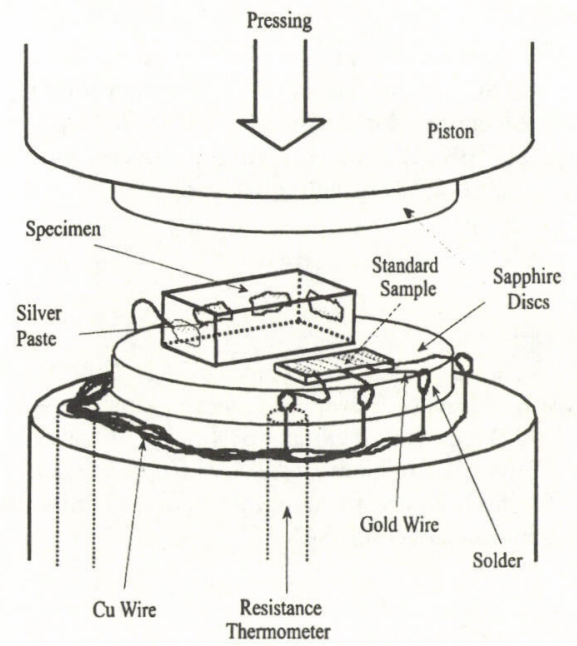

Fig.2. Arrangement of the specimen. Another one is a monitor of temperature using its $T_{c}$.

\subsection{AC Susceptibility}

AC susceptibility under uniaxial stress was measured with a standard lock-in technique. The coil system consists of primary and oppositely wounded secondary coils (Fig.3). AC magnetic field with the amplitude of $0.69 \mathrm{Oe}$ and the frequency of $3 \mathrm{kHz}$ was used. The coil system is surrounded by two copper tubes as shown in the figure. In the measurement, the specimen was cooled down to $77 \mathrm{~K}$ under zero magnetic field after increasing the stress at room temperature.

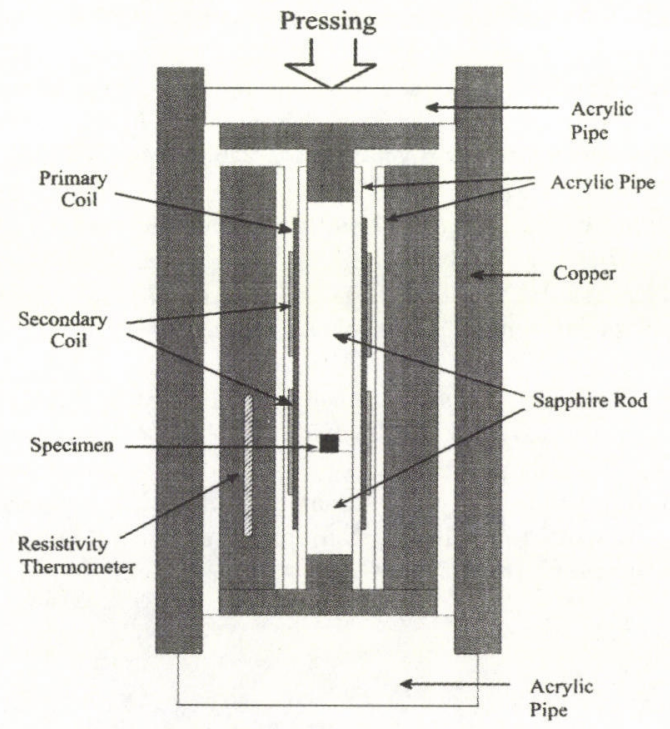

Fig.3. Scheme of apparatus for AC susceptibility under uniaxial stress.

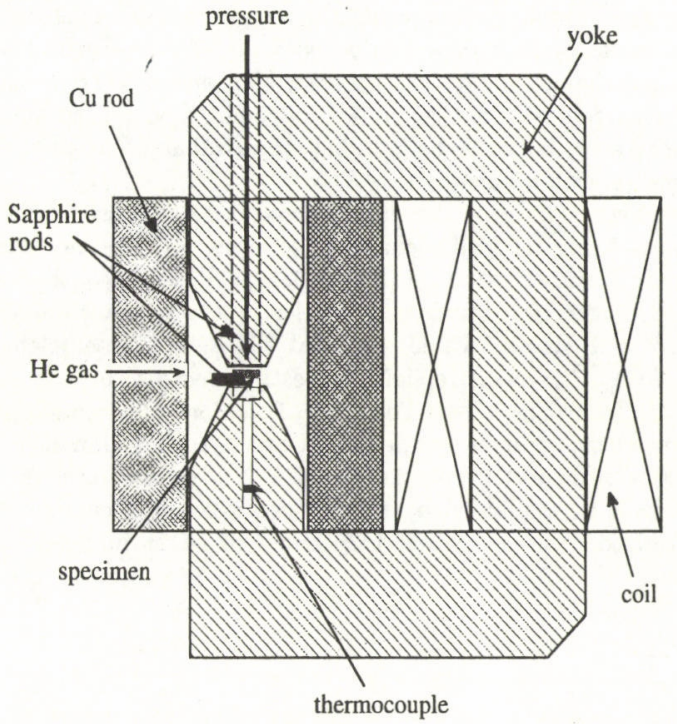

Fig.4. Scheme of apparatus for Hall effect under uniaxial stress.

\subsection{Hall Effect}

The Hall coefficient under hydrostatic stress is measured using the apparatus shown in Fig.4. The uniaxial stress is applied with sapphire rods as shown in the figure. Hall voltage is measured using a double-AC technique under maximum field of $0.3 \mathrm{~T}(2 \mathrm{~Hz})$ and current of $30 \mathrm{~mA}(5 \mathrm{~Hz})$. The technique allows us to measure small signal $(7 \mathrm{~Hz})$ of Hall voltage by using a spectrum analyzer, even though superconducting magnet is not used. The uniformity of the magnetic field at the specimen is order of $5 \%$ or so. The system in the figure is cooled down to $77 \mathrm{~K}$ in zero field, then the field was applied.

A single crystal of $\mathrm{Bi}_{2} \mathrm{Sr}_{2} \mathrm{CaCu}_{2} \mathrm{O}_{x}$ with the size of $6 \mathrm{~mm}$ in diameter was used for the study. It was carefully cleaved and cut into several pieces with a wire saw. The crystal was confirmed to be in under-doped state. The dependence of $T_{c}$ of a specimen on hydrostatic stress $\left(P_{\text {hydro }}\right)$ was measured up to $1 \mathrm{GPa}$, then measurements under uniaxial stress on it were followed.

\section{Results and Discussions}

From the measurements under hydrostatic stress, it was confirmed that $T_{c}$ increased with a coefficient of $d T_{c} / d P_{\text {hydro }} \simeq$ $+2.0 \mathrm{~K} / \mathrm{GPa}$. All of our specimens from the crystal showed the same behavior of positive sign of $d T_{c} / d P_{\text {hydro. }}$. This behavior is explained by a simple pressure induced charge transfer model (PICT-model) as in YBCO system. The model suggests that stress induced change of transport properties can be explained by the change of the carrier density in $\mathrm{CuO}_{2}$ planes. Though the model is not appropriate for several systems[9][10], the increase in $T_{c}$ by hydrostatic stress of our specimen is explained by the model due to the increase of carrier (hole) from $\mathrm{BiO}$ plane. 
On the contrary to the result, the measurements under uniaxial stress on electrical conductivity and AC susceptibility revealed that $d T_{c} / d P_{\| c}$ has negative sign and $d T_{c} / d P_{\perp c}$ has positive one[8]. From the simple model $d T_{c} / d P_{\| c}$ should have positive sign, since the distance between $\mathrm{BiO}$ and $\mathrm{CuO}_{2}$ plane decreases with uniaxial stress $P_{\| c}$.

Hall effect is one of the effective methods to observe the change of carrier. Hall coefficient decreases with the increase of hydrostatic stress (Fig.5(a)), which is consistent with the model, suggesting increase of carrier in $\mathrm{CuO}_{2}$ plane under hydrostatic stress. Under uniaxial stress, Hall coefficient, $d R_{H} / d P_{\| c}$, has positive sign, suggesting decrease of hole in $\mathrm{CuO}_{2}$ plane (Fig.5(b)). Therefore, it is concluded that the simple charge transfer model is not enough to explain stress effect in transport properties in Bi2212. Probably these results will be explained by taking into account a change of hybridized orbitals of $\mathrm{Bi} 6 p-\mathrm{O} 2 p$ and $\mathrm{Cu} 3 d-\mathrm{O} 2 p$ induced by stress.

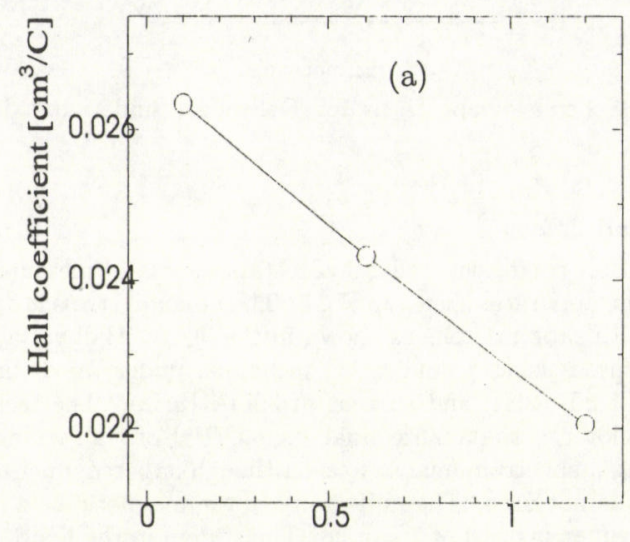

Hydrostatic stress $[G P a]$

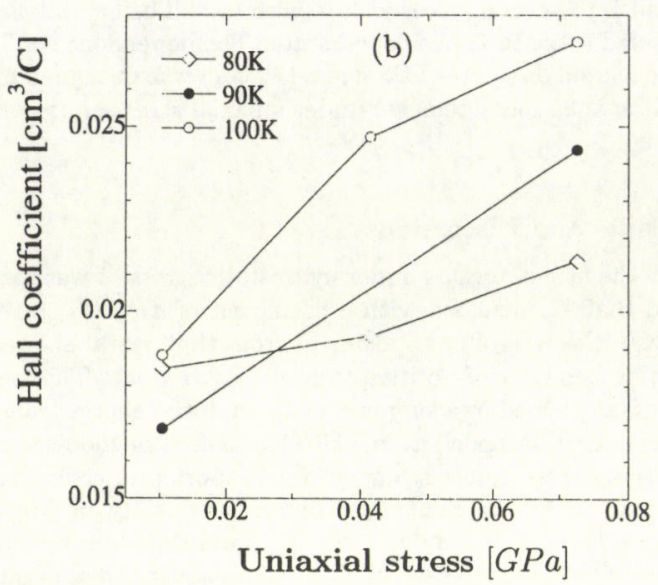

Fig.5. Dependence of Hall coefficient on hydrostatic(a) and on uniaxial stress(b).

\section{Conclusion}

The measuring systems of transport properties under uniaxial stress on HTSC are presented. The accuracy of them are evaluated to be enough for the study on HTSC. Applying them to underdoped $\mathrm{Bi} 2212$ single crystal, unexpected results $\left(d T_{c} / d P_{\| c}<0, d T_{c} / d P_{\perp c}>0\right)$ were obtained.

\section{Acknowledgments}

The authors would like to express their thanks to A.M.Balbashov of Moscow Power Engineering Institute for providing us Bi-2212 crystal, thanks to N.Môri of Institute for Solid State Physics, University of Tokyo for providing us an apparatus for hydrostatic measurement, and thanks are also due to T.Yonezawa of Shinko-sha Co. for providing us a set of sapphire rods.

\section{References}

[1] L.Gao, Y.Y.Xue, F.Chen, Q.Xiong, R.L.Meng, D.Ramirez, C.W.Chu, J.H.Eggert and H.K.Mao: Phys.Rev., B 50:6, 4260 (1994)

[2] Y.Motoi, K.Fujimoto, H.Uwe and T.Sakudo: J.Phys.Soc.Jpn., 60-3, 384 (1991)

[3] F.Gugenberger, C.Mengast, G.Roth, K.Grube, V.Breit, T.Weber, H.W ühl, S.Uchida and Y.Nakamura: Phys.Rev., B 49, 13137 (1994)

[4] U.Welp, M.Grimsditch, S.Fleshlev, W.Nwssler, J.Downey,G.W.Crabtree and J.Guimpel: Phys.Rev.Lett., 64-14, 2130 (1992)

[5] T.Nakanishi, K.Fukamachi, N.Watanabe, K.Tsushima, A.M.Balbashov, C.Murayama and N.Môri: Proc. of 6th International Symposium on Superconductivity, 363 (1993)

[6] K.Fukamachi, Y.Ueda, N.Watanabe, K.Tsushima, A.M.Balbashov and N.Môri: Proc. of 7th International Symposium on Superconductivity, 225 (1994)

[7] Y.Ueda, H.Tsunematsu, N.Watanabe, K.Tsushima and N.Môri: Proc. of 8th International Symposium on Superconductivity, 73 (1995)

[8] N.Watanabe, Y.Ueda, K.Tsushima and N.Môri: Proc. of 9th International Symposium on Superconductivity 85 (1996)

[9] N.Tanahashi, Y.Iye, T.Tamegai, C.Murayama, N.Môri, S.Yomo, N.Okazaki and K.Kitazawa: Jpn.J.Appl.Phys., 28, 762 (1989)

[10] C.Murayama, Y.Iye, T.Enomoto, N.Môri, Y.Yamada, T.Matsumoto, Y.Kubo, Y.Shimakawa and T.Manako: Physica, C 183, 277 (1991) 